\section{Correspondence on '2018 update of the EULAR recommendations for the management of hand osteoarthritis'}

We were inspired by reading the article entitled '2018 update of the EULAR recommendations for the management of hand osteoarthritis' by Kloppenburg et al. ${ }^{1}$ This up-to-date guideline will inform all health professionals and patients about optimal management by shared decision-making and may help advance research of hand osteoarthritis (OA). In overarching principles, this recommendation highlighted that the primary goal of managing hand $\mathrm{OA}$ is to control pain and stiffness and to optimise hand function and quality of life. This guideline also emphasised that management of hand OA should be individualised, taking into account its localisation and severity as well as comorbidities. The comorbidities should be thoroughly evaluated for individualised management of hand OA. Particularly, systemic diseases such as cardiac disease and/or hypertension, diabetes and depression are commonly accompanied with OA. ${ }^{2}$ Apart from systemic diseases, various localised upper extremity musculoskeletal diseases (UEMDs) also contribute to greater pain and poor performance-based physical function in people with hand OA. However, there have been few small studies in which carpal tunnel syndrome (CTS) or rotator cuff tear (RCT) has been commonly associated with hand OA. ${ }^{3}{ }^{4}$ Moreover, no study has examined the effects of accompanying UEMDs on hand pain and function in patients with hand OA. Hence, we comprehensively investigated the influence of various UEMDs on hand pain and functional status in patients with hand OA.

Overall, 311 patients with hand OA participated in this study. All patients underwent physical examination, plain radiography of both hands, MRI of both shoulders and nerve conduction velocity (NCV) examinations of both median nerves to evaluate accompanying UEMDs. Patients also answered the Korean version of the Australian/Canadian (AUSCAN) Osteoarthritis Hand Index and the Patient Health Questionnaire-2 (PHQ-2). The Neck Disability Index (NDI) and disabilities of the arm, shoulder and hand (DASH) scores were also assessed to investigate pain and function of upper extremities. Hand OA diagnoses adhered to the 1990 American College of Rheumatology classification criteria. CTS was confirmed by NCV findings, and RCT was diagnosed by means of MRI findings. Myofascial pain syndrome (MPS) was diagnosed by palpations of myofascial trigger points. Medial and lateral epicondylitis were defined by pain in the epicondyle region, which was provoked by resistance of either the extensor or flexor muscles of the wrist. Patients or the public were not involved in the design, or conduct, or reporting or dissemination plans of our research.

There were 215 women (69.1\%) and median age was 61.0 (IQR 56.0-67.0) years. The number of patients with accompanying MPS, CTS, lateral epicondylitis, medial epicondylitis and RCT were 249 (80.1\%), 151 (48.6\%), 134 (43.1\%), 79 (25.4\%) and 192 (61.7\%), respectively. Analyses were performed using the generalised linear model to determine whether hand pain and function (AUSCAN score) were affected by the presence of each UEMD. In bivariate analysis, women, depression (PHQ-2 $\geq 3$ ) and accompanying MPS showed high AUSCAN pain, stiffness, disability and total scores. After controlling for confounding variables, we obtained the following results (figure 1). We found that only MPS increased the total AUSCAN score (exp(B) (estimated OR) 1.789; 95\% CI 1.121 to $2.856, \mathrm{p}=0.015$ ), which significantly affected the AUSCAN

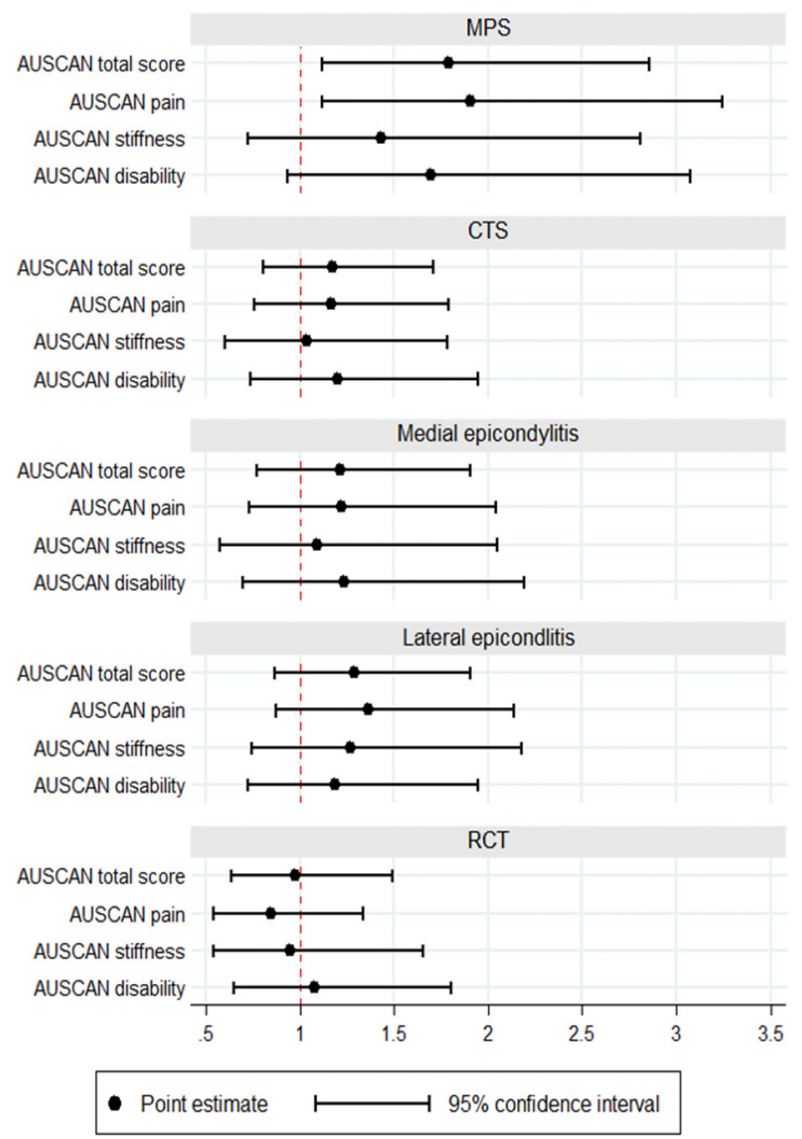

Figure 1 The effect of concomitant upper extremity musculoskeletal diseases on pain and functional status of patients with hand osteoarthritis. $p$ value by generalised linear model with gamma distribution and log link function. Adjusted by variables which is $p$ value $<0.05$ in bivariate analysis for each model (age, sex, depression). AUSCAN, the Australian/Canadian Osteoarthritis Hand Index; MFPS, myofascial pain syndrome; CTS, carpal tunnel syndrome; RCT, rotator cuff tear.

pain $(\exp (B) 1.907 ; 95 \%$ CI 1.121 to $3.246, p=0.017)$. CTS, RCT, lateral and medial epicondylitis had no significant effects on AUSCAN scores.

There are two possible reasons why MPS affects hand pain in patients with hand OA. First, central sensitisation of MPS may affect hand pain in patients with hand OA ${ }^{5}$ The relative ischaemia of skeletal muscle would cause elevated levels of sensitising substances and lead to central sensitisation. As a result, the pain threshold is lowered, and pain hypersensitivity is produced in patients with MPS. In this study, other assessment scores of upper extremity pain were significantly increased in patients with MPS -NDI pain scores $(\exp (B) 1.912$ CI 1.063 to 3.440, $\mathrm{p}=0.030)$ and DASH symptom scores $(\exp (\mathrm{B}) 1.607 \mathrm{CI} 1.165$ to $2.215, \mathrm{p}=0.004)$. Second, the nature of referred pain may affect hand pain in patients with hand OA. MPS produces hyperirritable nodules within taut bands of skeletal muscle known as myofascial trigger points and palpations of these nodules can produce referred pain. ${ }^{6}$

In conclusion, MPS is a common complication and has a significant impact on hand pain in patients with hand OA. Therefore, accompanying MPS needs to be addressed and managed to improve hand pain and function in patients with hand OA. 
Young Sun Suh, ${ }^{1,2}$ Hyun-Ok Kim, ${ }^{1,2}$ Chang Han Lee, ${ }^{3,4}$ Chul Ho Yoon, ${ }^{3,4}$ Yun-Hong Cheon $\circledast^{1,5}$ Mingyo Kim, ${ }^{1,5}$ Hanna Lee, ${ }^{1,5}$ Sang-II Lee $\overbrace{}^{1,5,6}$

${ }^{1}$ Rheumatology, Internal Medicine, Gyeongsang National University College of Medicine, Jinju, Gyeongsangnam-do, Republic of Korea

${ }^{2}$ Rheumatology, Internal Medicine, Gyeongsang National University Changwon Hospital, Changwon, Gyeongsangnam-do, Republic of Korea

${ }^{3}$ Rehabilitation Medicine, Gyeongsang National University Hospital, Jinju,

Gyeongsangnam-do, Republic of Korea

${ }^{4}$ Rehabilitation Medicine, Gyeongsang National University College of Medicine, Jinju, Gyeongsangnam-do, Republic of Korea

${ }^{5}$ Rheumatology, Internal Medicine, Gyeongsang National University Hospital, Jinju, Gyeongsangnam-do, Republic of Korea

${ }^{6}$ Internal Medicine and Institute of Health Science, Gyeongsang National University College of Medicine, Jinju, Republic of Korea

Correspondence to Professor Sang-II Lee, Internal Medicine and Institute of Health Science, Gyeongsang National University College of Medicine, Jinju 660-702, Korea (the Republic of); goldgu@gnu.ac.kr

Contributors Conception of the work: YSS, H-OK, S-IL. Acquisition of data: YSS, $\mathrm{H}-\mathrm{OK}, \mathrm{CHL}, \mathrm{CHY}, \mathrm{YHC}, \mathrm{MK}, \mathrm{HL}$. Analysis or interpretation of data: YSS, H-OK, S-IL. Drafting: YSS, H-OK. Revision of the work: YSS, S-IL. All authors read and approved the final manuscript.

Funding This work was supported by grants (NRF-2015R1A5A2008833) from the National Research Foundation of Korea (NRF) funded by the Korean government (http://www.nrf.re.kr/index) and the Centre for Farmer's Safety and Health, Ministry of Agriculture, Food and Rural Affairs, Republic of Korea.

Competing interests None declared.

Patient and public involvement Patients and/or the public were not involved in the design, or conduct, or reporting, or dissemination plans of this research.

Patient consent for publication Not required.

Ethics approval The Institutional Review Board of the Gyeongsang National University Hospital approved this study (GNUH 2015-02-001).
Provenance and peer review Not commissioned; internally peer reviewed.

(c) Author(s) (or their employer(s)) 2020. No commercial re-use. See rights and permissions. Published by BMJ.

YSS and H-OK contributed equally.

$$
\text { Check for updates }
$$

To cite Suh YS, Kim H-O, Lee CH, et al. Ann Rheum Dis Epub ahead of print: [please include Day Month Year]. doi:10.1136/annrheumdis-2020-218887

Received 18 August 2020

Accepted 19 August 2020

Ann Rheum Dis 2020;0:1-2. doi:10.1136/annrheumdis-2020-218887

\section{ORCID iDs}

Yun-Hong Cheon http://orcid.org/0000-0002-0099-6253

Sang-II Lee http://orcid.org/0000-0002-8283-7001

\section{REFERENCES}

1 Kloppenburg M, Kroon FP, Blanco FJ, et al. 2018 update of the EULAR recommendations for the management of hand osteoarthritis. Ann Rheum Dis 2019;78:16-24.

2 Marshall M, Watt FE, Vincent TL, et al. Hand osteoarthritis: clinical phenotypes, molecular mechanisms and disease management. Nat Rev Rheumatol 2018:14:641-56

3 Shiri R. Arthritis as a risk factor for carpal tunnel syndrome: a meta-analysis. Scand J Rheumatol 2016:45:339-46.

4 Suh YS, Kim H-O, Cheon Y-H, et al. Metabolic and inflammatory links to rotator cuff tear in hand osteoarthritis: a cross sectional study. PLoS One 2020;15:e0228779.

5 Fernández-de-las-Peñas C, Dommerholt J. Myofascial trigger points: peripheral or central phenomenon? Curr Rheumatol Rep 2014;16:395.

6 Gerwin RD. Myofascial pain syndromes in the upper extremity. J Hand Ther 1997;10:130-6. 